\title{
Anomalous Dimensions of Twist-Two Operators with High Lorentz Spin
}

\author{
B. Basso * \\ Laboratoire de Physique Théorique - Université de Paris XI \\ 91405 ORSAY CEDEX - FRANCE
}

\begin{abstract}
Twist-two anomalous dimensions depend on their Lorentz spin and admit an asymptotic expansion for large values of this quantum number. Recent multi-loop QCD calculations of twist-two anomalous dimensions revealed the existence of an intriguing feature : The coefficients parameterizing their large Lorentz spin expansion satisfy an infinite system of equations, and so are not all independent. We argue that these relations follow from the parity preserving property of some related function, the scaling function.
\end{abstract}

\section{Introduction}

Forward matrix elements of twist-two Wilson operators, $\left\langle O_{N}^{(a)}(0)\right\rangle$, provide the dominant contribution to the structure functions of deeply inelastic scattering in the limit of large momentum transfer. They carry Lorentz spin $N$ and some additional quantum numbers (a). The twist-two operators with the same Lorentz spin mix under a change of the renormalization scale $\mu$ and satisfy the Callan-Symanzik equation

$$
\left[\mu \frac{\partial}{\partial \mu}+\beta\left(\alpha_{s}\right) \frac{\partial}{\partial \alpha_{s}}\right]\left\langle O_{N}^{(a)}(0)\right\rangle=-\gamma^{a b}\left(N, \alpha_{s}\right)\left\langle O_{N}^{(b)}(0)\right\rangle .
$$

The mixing matrix $\gamma\left(N, \alpha_{s}\right)$, entering Eq. (1), depends on the Lorentz spin and admits a perturbative expansion in the strong coupling constant $\alpha_{s}$

$$
\gamma\left(N, \alpha_{s}\right) \equiv \gamma(N)=\frac{\alpha_{s}}{2 \pi} \gamma_{1}(N)+\frac{\alpha_{s}^{2}}{4 \pi^{2}} \gamma_{2}(N)+\ldots
$$

The size of the mixing matrix does not depend on the Lorentz spin, but on some other quantum numbers of the Wilson operators under consideration. For the sake of clarity, we will consider two particular examples of twist-two mixing matrices : quark non-singlet anomalous dimension and singlet anomalous dimensions matrix

$$
\gamma^{n s}(N), \quad \gamma^{s}(N)=\left(\begin{array}{cc}
\gamma^{q q}(N) & \gamma^{q g}(N) \\
\gamma^{g q}(N) & \gamma^{g g}(N)
\end{array}\right)
$$

computed in $\overline{M S}$-scheme.

Beyond the leading order, the analytic expressions for $\gamma^{n s}(N)$ and $\gamma^{s}(N)$ are very cumbersome. Their large Lorentz spin expansions are however simpler and exhibit interesting features. In particular, both quark non-singlet and diagonal elements of the singlet mixing matrix are known to scale logarithmically with the Lorentz spin [3]

$$
\gamma(N)=A\left(\alpha_{s}\right) \ln N+\mathcal{O}\left(N^{0}\right),
$$

*Based on a work done in collaboration with G. P. Korchemsky [2]. 
where $A\left(\alpha_{s}\right) / 2=\Gamma_{\text {cusp }}\left(\alpha_{s}\right)$ is the universal cusp anomalous dimension. It only depends on the colour charge (quadratic Casimir) of the fundamental field entering the Wilson operator.

Subleading corrections, suppressed by powers of $1 / N$, are not universal and depend on additional quantum numbers. For large $N$, it is convenient to parameterize them as follows

$$
\begin{gathered}
\gamma(N)=\frac{1}{2} A\left(\alpha_{s}\right) \ln \left[N(N+1) e^{2 \gamma_{E}}\right]+B\left(\alpha_{s}\right) \\
+\frac{1}{2} C\left(\alpha_{s}\right) N^{-1} \ln \left[N(N+1) e^{2 \gamma_{E}}\right]+D\left(\alpha_{s}\right) N^{-1}+\mathcal{O}\left(N^{-2} \ln ^{p} N\right) .
\end{gathered}
$$

Based on three-loop explicit expressions for anomalous dimensions, Moch, Vermaseren, Vogt [4] and Dokshitzer, Marchesini, Salam [5] observed interesting relations between the expansion coefficients $A, B, C, D$ : Both for $\gamma^{n s}(N)$ and for diagonal elements of $\gamma^{s}(N)$, they noted that ${ }^{\mathrm{a}}$

$$
C\left(\alpha_{s}\right)=\frac{1}{2} A^{2}\left(\alpha_{s}\right), \quad \text { and that } \quad D\left(\alpha_{s}\right)=\frac{1}{2} A\left(\alpha_{s}\right) B\left(\alpha_{s}\right)-\frac{1}{2} A\left(\alpha_{s}\right) \frac{\beta\left(\alpha_{s}\right)}{\alpha_{s}} .
$$

Equations (2) state that the expansion coefficients are not all independent. Moreover, the expressions for $C$ and $D$ to order $\mathcal{O}\left(\alpha_{s}^{n+1}\right)$ are determined by the coefficients $A$ and $B$ to order $\mathcal{O}\left(\alpha_{s}^{n}\right){ }^{\text {b }}$ In the next section, we shall see that Eqs. (2) belong to an infinite system of equations, relating the expansion coefficients of twist-two anomalous dimensions for large Lorentz spin. We shall argue that these equations follow from the parity preserving property of the scaling function.

\section{Parity preserving property of the scaling function}

In an attempt to interpret Eqs. (2), Dokshitzer, Marchesini and Salam [5] proposed to define the scaling function $f$ through the relation

$$
\gamma(N)=f\left(N+\frac{1}{2} \gamma(N)\right) .
$$

Then, they observed that, neglecting the beta-function contribution to $D$, Eqs. (2) are equivalent to the vanishing of corrections $\propto N^{-1}$ in the following parameterization of the scaling function

$$
f(N)=\frac{1}{2} A\left(\alpha_{s}\right) \ln \left[N(N+1) e^{2 \gamma_{E}}\right]+B\left(\alpha_{s}\right)+0 . N^{-1}+\mathcal{O}\left(N^{-2} \ln ^{p} N\right),
$$

for large $N$. In their approach, the parameterization, Eq. (4), and the vanishing of the correction $\propto N^{-1}$ were motivated by some physical arguments. We would like to indicate another point of view with help of an explicit example. As was already mentioned, Eqs. (3) and (4) fail to take into account the beta-function contribution. To circumvent this difficulty we shall below interpret Eq. (3) in the framework of conformal symmetry and modify it in order to account for the non-vanishing beta-function. But first, let us examine the example

aTo two-loop accuracy, the relation between $A$ and $C$ was first observed by Curci, Furmanski and Petronzio [6] for the quark non-singlet anomalous dimension.

b Taking account that $\beta\left(\alpha_{s}\right)=\mathcal{O}\left(\alpha_{s}^{2}\right)$. 
of the quark non-singlet anomalous dimension. To one-loop order, the anomalous dimension is not sensitive to the beta-function contribution and is given by

$$
\gamma(N)=\frac{\alpha_{s}}{2 \pi} \gamma_{0}(N)+\mathcal{O}\left(\alpha_{s}^{2}\right), \quad \gamma_{0}(N)=C_{F}\left[4 \psi(N+1)+4 \gamma_{E}-3-\frac{2}{N(N+1)}\right] .
$$

To lowest order, Eq. (3) translates into

$$
f(N)=\gamma(N)+\mathcal{O}\left(\alpha_{s}^{2}\right)=\frac{\alpha_{s}}{2 \pi} \gamma_{0}(N)+\mathcal{O}\left(\alpha_{s}^{2}\right)
$$

Hence

$$
f(N)=\frac{\alpha_{s}}{2 \pi} C_{F}\left[4 \psi(N+1)+4 \gamma_{E}-3-\frac{2}{N(N+1)}\right]+\mathcal{O}\left(\alpha_{s}^{2}\right)
$$

The large $N$ expansion of $\psi(N+1)$ only runs in negative integer powers of the parameter $N(N+1)$

$$
\psi(N+1)=\frac{1}{2} \ln [N(N+1)]+\frac{1}{6} \frac{1}{N(N+1)}-\frac{1}{30} \frac{1}{N^{2}(N+1)^{2}}+\ldots+\mathcal{O}\left(N^{-n}(N+1)^{-n}\right),
$$

and from Eq. (5) we conclude that the large $N$ expansion of the scaling function has the same property. We would like to stress that it is this property that guarantees the absence of contributions $\propto N^{-1}$ in the large $N$ expansion of the scaling function, Eq. (4). To go beyond one-loop, we need to include into consideration the beta-function contribution.

The scaling function, as defined in Eq. (3), is tied to conformal symmetry. Indeed, if QCD were conformally invariant, the quantity $j=N+\gamma(N) / 2$ would be conformal spin and would be a good quantum number to classify Wilson operators according to representations of the conformal group. In this case, it would be natural to express anomalous dimensions as a function of the conformal spin $j$, rather than the Lorentz spin $N$. This immediately leads to Eq. $(3): \gamma(N)=f(j)$.

However, QCD is not a conformally invariant theory. Nevertheless, working within the $\overline{M S}$-scheme, it is possible to incorporate conformal symmetry breaking corrections and to arrive at the following improved definition of the scaling function [2]

$$
\gamma(N)=f\left(N+\frac{1}{2} \gamma(N)-\frac{\beta\left(\alpha_{s}\right)}{2 \alpha_{s}}\right) .
$$

Starting from this definition of the scaling function, the absence of contributions $\propto N^{-1}$ in the large $N$ expansion of the scaling function, Eq. (4), leads to Eqs. (2), including the beta-function contribution.

The one-loop non-singlet anomalous dimension considered above suggests that the scaling function possesses an important property : The large $N$ expansion of the scaling function only runs in negative integer powers of the parameter $J^{2}=N(N+1)$

$$
f(N)=\frac{1}{2} A\left(\alpha_{s}\right) \ln \left[J^{2} e^{2 \gamma_{E}}\right]+B\left(\alpha_{s}\right)+f^{(1)}\left(\ln J^{2}\right) J^{-2}+f^{(2)}\left(\ln J^{2}\right) J^{-4}+\mathcal{O}\left(J^{-6}\right) .
$$

and so is invariant under the parity $N \rightarrow-N-1$. We refer to this relation as the parity preserving property. 
We verified [2] that the parity preserving property holds in QCD for ${ }^{\mathrm{C}}$

- two-loop longitudinally polarized singlet anomalous dimensions,

- two-loop gluon linearly polarized anomalous dimension,

- two-loop quark transversity anomalous dimension,

- three-loop non-singlet unpolarized anomalous dimension,

- three-loop singlet unpolarized anomalous dimensions.

Replacing the scaling function in Eq. (6) by its expression, Eq. (7), and expanding $\gamma(N)$ in powers of $1 / J$, one obtains that the coefficients in front of odd powers of $1 / J$ can be expressed in terms of the coefficients accompanying even powers of $1 / J$ to less number of loops. The resulting infinite system of equations include in particular Eqs. (2).

\section{Conclusion and outlooks}

Starting from the observation that twist-two anomalous dimensions fulfill some intriguing relations, we identified them as a manifestation of the parity preserving property of some related function, the scaling function. Using results for the anomalous dimensions available in the literature, we verified that this property holds in QCD and that it leads to an infinite system of equations constraining twist-two anomalous dimensions.

The parity preserving property is a robust phenomenon : it holds in QCD to all loops in the large $\beta_{0}$ limit for both singlet and non-singlet anomalous dimensions. It can also be extended to a subclass of higher twist operators. It would be interesting to test the parity preserving property for these operators. However, the origin of this property still remains to be understood.

\section{Acknowledgments}

I would like to thank the organizers of DIS 2007 for hospitality. The work was supported in part by the French Agence Nationale de la Recherche under grant ANR-06-BLAN-0142-02.

\section{Bibliography}

\section{References}

[1] Slides:

http: //indico. cern. ch/contributionDisplay $\cdot$ py? contribId=183\&sessionId=8\& conf Id=9499

[2] B. Basso and G. P. Korchemsky, Nucl. Phys. B 775, 1 (2007).

[3] G. P. Korchemsky, Mod. Phys. Lett. A 4, 1257 (1989); G. P. Korchemsky and G. Marchesini, Nucl. Phys. B 406, 225 (1993).

[4] S. Moch, J. A. M. Vermaseren and A. Vogt, Nucl. Phys. B 688, 101 (2004); A. Vogt, S. Moch and J. A. M. Vermaseren, Nucl. Phys. B 691, 129 (2004).

[5] Yu. L. Dokshitzer, G. Marchesini and G. P. Salam, Phys. Lett. B 634, 504 (2006).

[6] G. Curci, W. Furmanski and R. Petronzio, Nucl. Phys. B 175, 27 (1980).

${ }^{\mathrm{c}}$ Note that the parity preserving relation only holds for eigenvalues of the mixing matrix and not for each element independently. Note also that the explicit expression for the scaling function depends on the particular anomalous dimension we consider. 